\title{
Design of Music Spectrum Display Based on STC12C5A60S2
}

\author{
Lihui Sun ${ }^{1,}$, Jun $\mathrm{Yu}^{2}$, b \\ ${ }^{1}$ School of Information and Control Engineering, Jilin Institute of Chemical Technology, Jilin \\ 132022, China \\ 2Dean`s Office, Jilin Institute of Chemical Technology, Jilin 132022, China \\ asunlihui2000@126.com, b83770573@qq.com
}

\begin{abstract}
Most of audio players on the market use the hardware filter or DSP chip to process spectrum, the use of the techniques on the one hand requires high hardware costs, on the other hand, software and hardware implementations is more complex. In order to overcome the shortcomings, a spectrum display using fast Fu Liye transform (FFT) algorithm is designed. The audio file in SD card is decoded through STC12C5A60S2 microcontroller, and the FFT algorithm is used to sample, quantify and divide the audio signal through $A / D$ internal microcontroller, the results being processed is sent to the LED dot matrix display screen, in addition, the system is introduced into the sensor, and the automatic adjustment of the display brightness is realized. This template explains and demonstrates how to prepare your camera-ready paper for Trans Tech Publications. The best is to read these instructions and follow the outline of this text.
\end{abstract}

Keywords: Music, STC12C5A60S2, Fu Liye transform.

\section{Introduction}

With the improvement of living standards, people not only pay attention to in the pursuit of quality, also pay more and more attention to the visual enjoyment, and spectral music show sound system considerably. At present, some spectrum display is completed by using hardware [1], some through the DSP processing and then display [2-6]. However, these display needs to achieve higher hardware, at the same time, computing speed will slow down. In view of the above shortcomings, the use of fast $\mathrm{Fu}$ Liye transforms (FFT) method is proposed to realize the music spectrum real-time display. The design not only to obtain the high-quality sound and clear display the process of audio, which give people on the visual aesthetics and the double enjoyment.

\section{System Composition}

Audio files in the SD card is decoded by the MP3 decoders, the audio will be restored by means of the audio signal being sent to the power amplifier circuit, at the same time, the spectrum display can use infrared remote control to realize wireless control. By using the A/D converter input audio signal sampling, after the fast Fu Liye transform algorithm (FFT) transform, take some of the frequency of the magnitude of the display, so as to drive the LED dot matrix screen lit the corresponding LED lights. At the same time, the display screen can realize the brightness adjustment function through the light sensitive circuit. The overall block diagram of the system is shown in figure 1. 


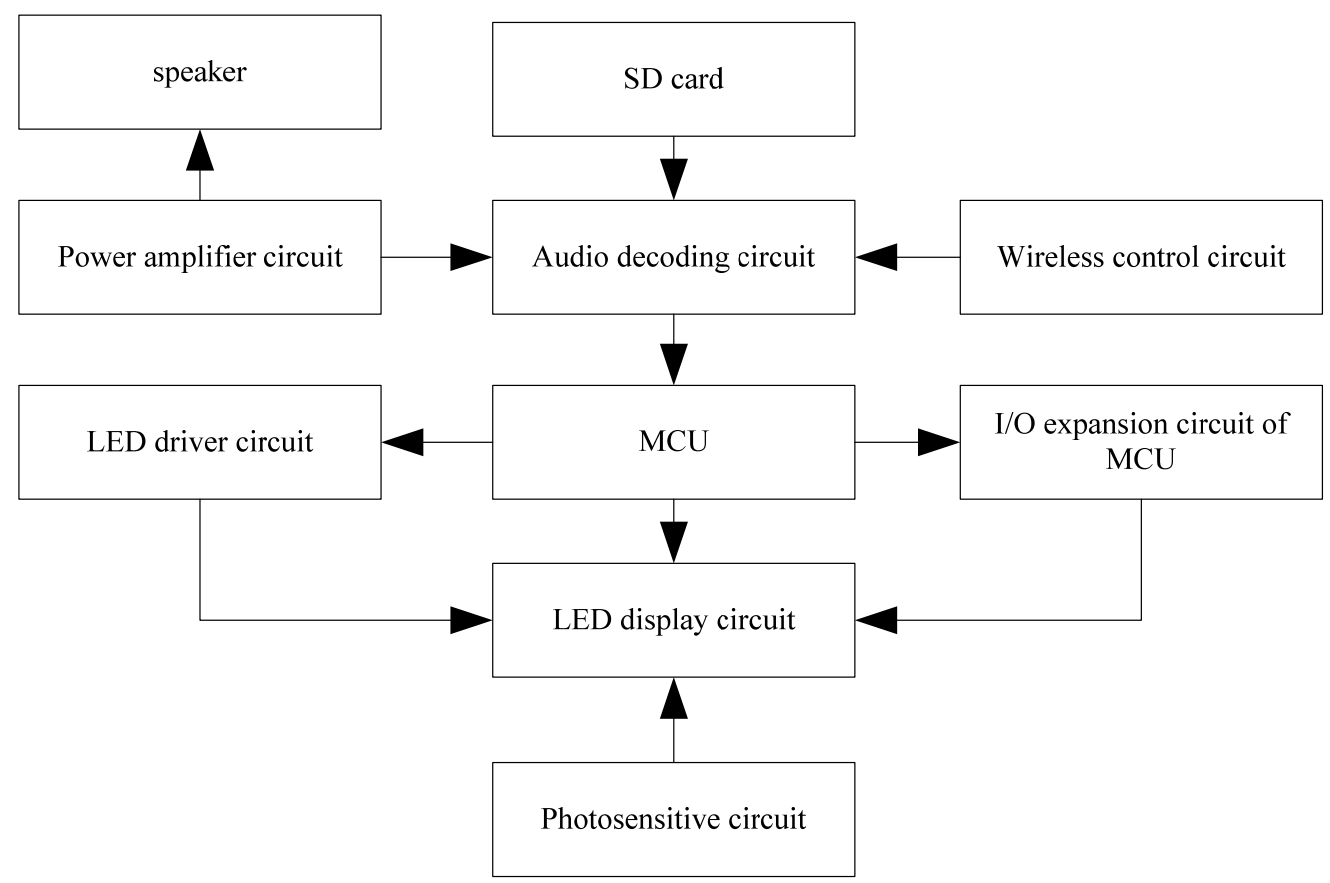

Figure 1. structure block diagram of music spectrum display system

\section{Principle of Spectrum Analysis}

FFT is the main means of spectral analysis. By using the periodicity and symmetry of trigonometric function, the DFT of the longer sequence is decomposed into shorter DFT, which reduces the computation workload. By the method, the number of complex multiplications of Fu Liye transformation can be reduced from $N^{2}$ to $N \log _{2}^{N} / 2$. Fast Fu Liye transform is mainly divided into two categories, a class is suitable for $\mathrm{N}$ for 2 of the integer powers of the operation, such as the basis of 2 algorithm, based on the 4 algorithms, the other is the $\mathrm{N}$ does not equal to 2 of the integer powers of the algorithm, such as hybrid algorithm.

The base 2-FFT algorithm is mainly composed of inversion sequence operation and multi stage butterfly operation. Inverted order operation is to store the raw data in the form of inverted order. After the operation, it can be output in normal order. According to the sample size, in the code segment system establish reverse table array, and then through the way of looking up table to realize fast reverse operation of the array, this operation and shift operation compared, computing speed get greatly improved. Butterfly algorithm is the idea of the FFT point $\mathrm{N}$ operation is divided into stages, each level has a butterfly operation, butterfly operation flow chart as shown in figure 2 .

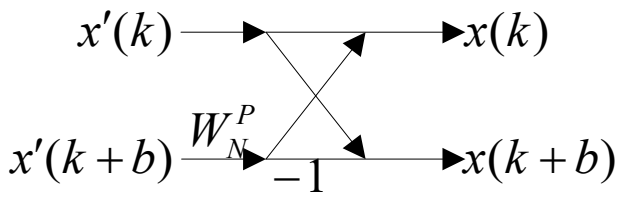

Figure 2. butterfly operation process

The corresponding relations are as follows:

$$
\begin{aligned}
& x(k)=x^{\prime}(k)+x^{\prime}(k+b) W_{N}^{P} \\
& x(k+b)=x^{\prime}(k)-x^{\prime}(k+b) W_{N}^{P}
\end{aligned}
$$

Among, $W_{N}^{P}=\cos \frac{2 \pi P}{N}-j \sin \frac{2 \pi P}{N}, \mathrm{P}=0,1, \cdots, \frac{N}{2}-1, \mathrm{~N}=2^{M}$.

In simple terms, the essence of butterfly algorithm is to simplify the mathematical operation with a certain complexity to a number of simple mathematical operations. In single-chip microcomputer 
(SCM), the butterfly operation uses its own characteristics, and transforms the mathematical operation of complexity into the calculation formula (1) and the formula (2), which greatly reduces the number of operations, saves the computation time, improve the efficiency.

All the N points of the FFT computing are achieved by butterfly algorithm through 3 layer cycle. The query table method is used to achieve a single butterfly operation in the innermost layer, each level of $N / 2$ butterfly operation is completed in the middle layer cycle, $\log _{2}^{N}$ level butterfly operation is completed by the outermost loop. In each level, the innermost layer loop completes the $N / 2^{L}$ butterfly operation, and the middle layer cycle controls the innermost layer circulation $2^{L-1}$ times, therefore, when the middle layer cycle is completed, the $N / 2$ butterfly operation is carried out. In fact, the L level calculation is completed in the middle layer and the middle layer cycle, at the same time, $\log _{2}^{\mathrm{N}}$ level butterfly operation is finally completed by the outermost layer.

\section{Principle of Spectrum Display}

The audio signal spectrum is divided into 128 sections in the system, the first 16 sections of the spectrum is display through the LED dot matrix screen. In order to be able to display the audio signal on the dot matrix screen, the result of FFT operation needs to be converted to input to the display. For example, if it is complex result from an FFT operation of the $n$ points $(n>1)$, the mode value being divided by $2 / N$ is the signal amplitiude of the corresponding to the frequency, if $n=1$, the mode value divided by $\mathrm{N}$ is the point signal phase of the corresponding to the frequency, the final results are stored in an array.

\section{Audio Decoding and Control Circuit}

Audio file in SD card is decoded by using S-210 plate of Shinco company in the audio decoding and the control circuit, the external of S-210 board is connected with an infrared receiving head, and the wireless control is carried out by an infrared remote controller. The decode board using dedicated MP3 decoder chip is designed to accomplish through the construction of Rong decoding scheme, it can be directly output audio signal, and decode the audio file in the SD card, support multiple audio format file, the biggest support 32 GB SD card. In addition, the decoder board also has a memory function, that is, after the restart, playing the song track unchanged, which is mainly achieved through the AT24C02. Audio decoder and control circuit as shown in figure 3.

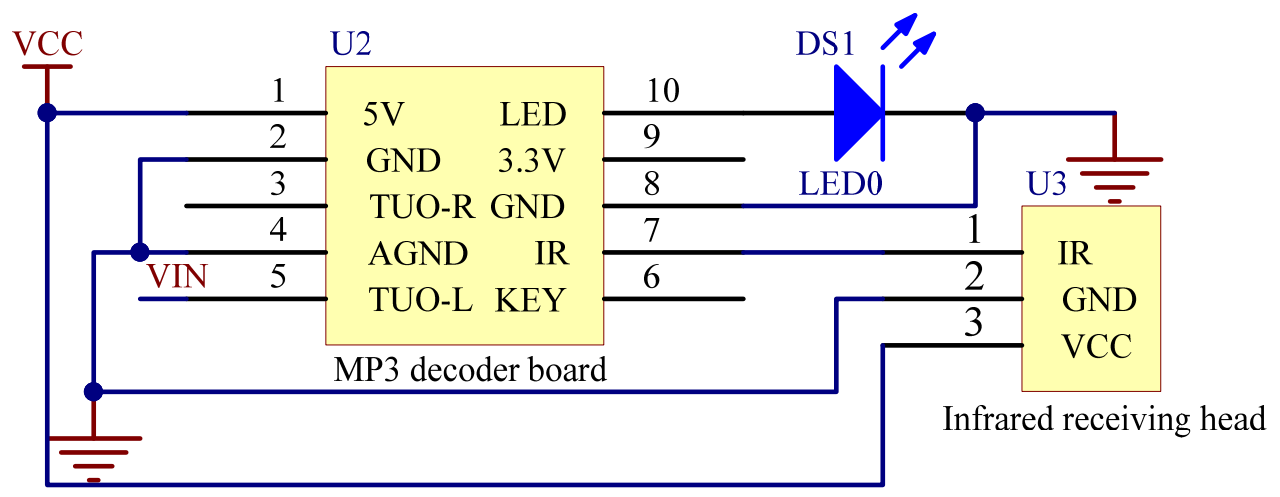

Figure 3. audio decoding and control circuit diagram

The infrared remote-control device in the system has the function of switch machine, the last one, the next song, pause, play, increase the volume, reduce the volume and other functions.

\section{Power Amplifier Circuit}

The music signal is amplified by power through the LM386 chip in the power amplifier circuit, the power amplification ratio is about 20 times, and the amplifier circuit is shown in Figure 4, which 
pin 7 connected capacitors is decouple the filter, pin 5 connected resistance and capacitance composite impedance correction network, which offset inductance component in load and prevent the self-excitation of the circuit.
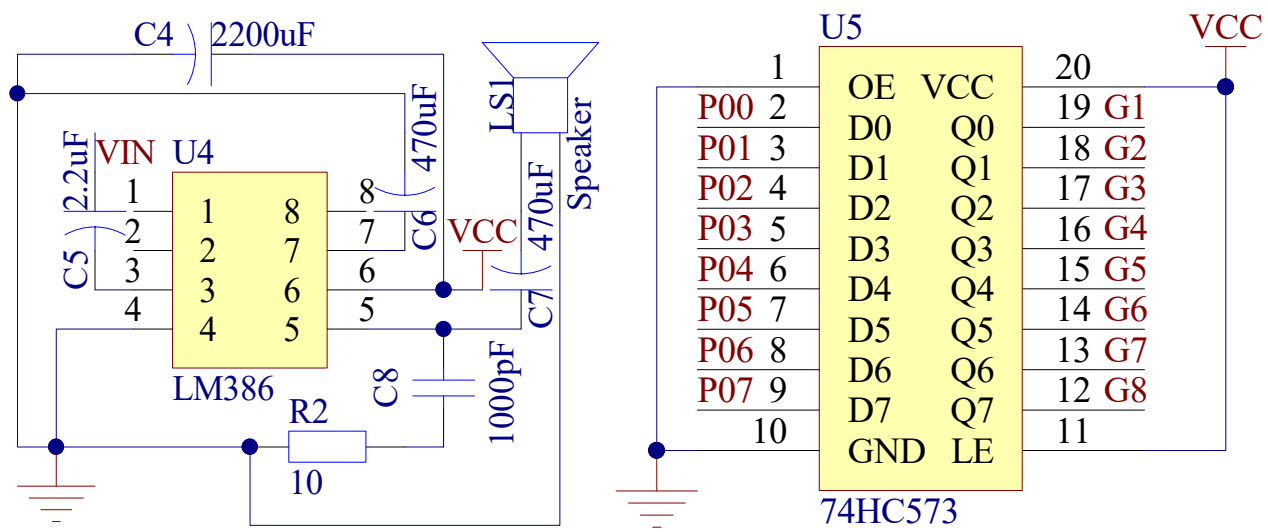

Figure 4. power amplifier circuit

Figure 5. LED dot matrix screen driver circuit

\section{Display Circuit}

$8 * 8$ dot matrix anode of $1588 \mathrm{BS}$ are spliced into $16 * 32$ dot matrix display in the system. Due to the lack of current driving ability of single chip I/O port, the row data of LED dot matrix screen is realized by 74HC573 chip, by connecting pin 1 and pin 11 of $74 \mathrm{HC} 573$ chip respectively to the ground and $+5 \mathrm{~V}$ power supply, it can make it have the output synchronization function, and the I/0 port can output $35 \mathrm{~mA}$ current. The photosensitive circuit is designed by the light sensitive resistor and the NPN transistor, which realize the function of automatic brightness adjustment. The LED drive circuit and the light sensing circuit are shown in Figure 5 and 6 respectively.

Because the system needs to use 32 column data to control the LED dot matrix screen, then the number of I/O port cannot meet the system requirements. So, through the application of $74 \mathrm{HC} 154$ chip to achieve the expansion of the I/O port, and then to achieve the LED dot matrix screen column data control. The I/O port expansion circuit diagram is shown in figure 7.

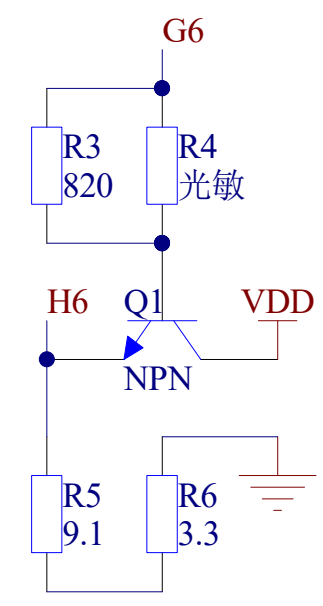

Figure 6. photo sensitive circuit

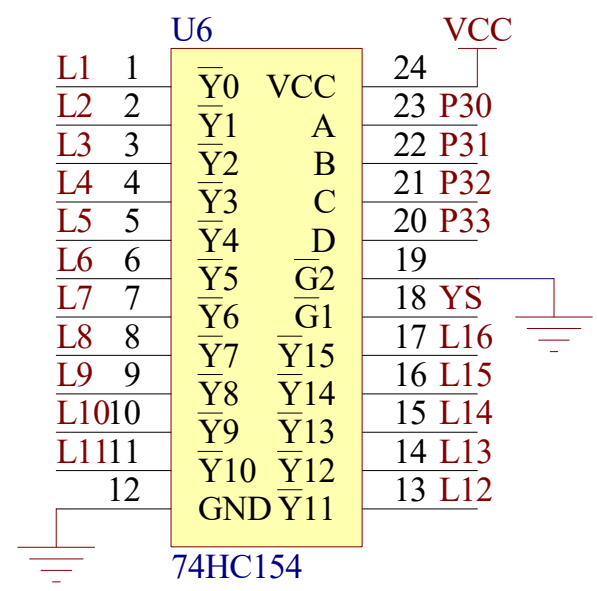

Figure 7. I/O port expansion circuit

\section{Software Design}

The software of this system mainly adopts the way of interruption. Using STC12C5A60S2 timer and interrupt resources, the system realizes the $\mathrm{A} / \mathrm{D}$ collection and processing, and realizes the dynamic display of the LED dot matrix display, the main program flow chart is shown in figure 8 . When the A/D acquisition related data 128 times, data will be processed in the A/D interrupt, data processing is mainly used by FFT. The operation of the FFT function is to transform the 128-point 
data, the FFT operation flow is shown in Figure 9. When the FFT operation is finished, the result is divided into sixteen frequency processing and sent to the LED display, LED dot matrix screen scanning is achieved in the timer interrupt.

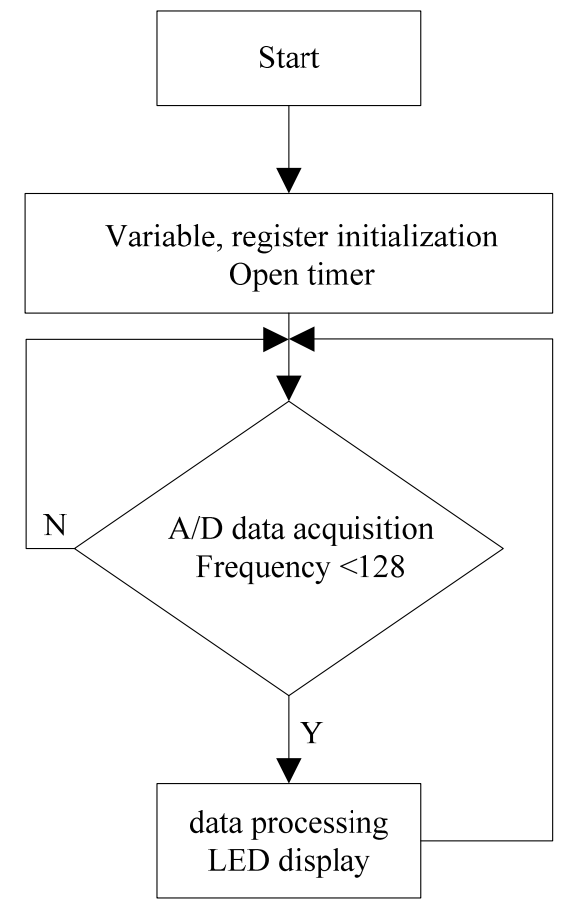

Figure 8. flow chart of main program

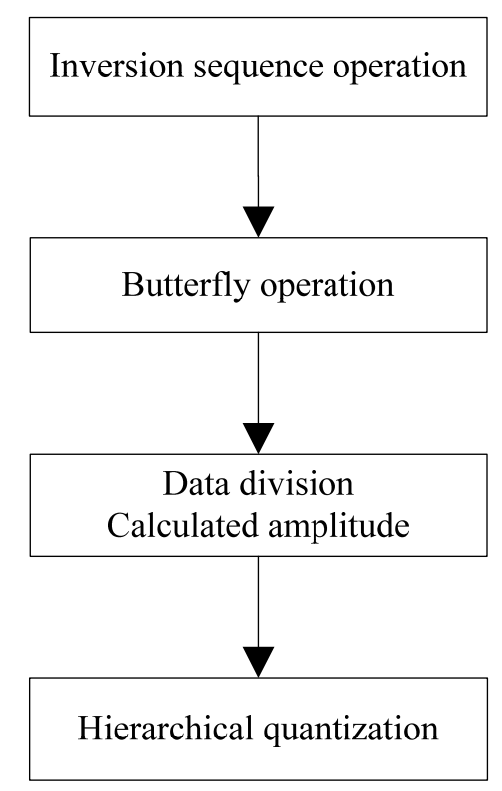

Figure 9. flow chart of FFT operation

\section{Text}

The main function of this system is embodied in the enhanced 51 MCU FFT processing and corresponding to the input data of the dot matrix screen and audio frequency spectrum and so on. The overall effect diagram of the system is shown in figure 10, shown in the figure is thousands of songs audio effects.

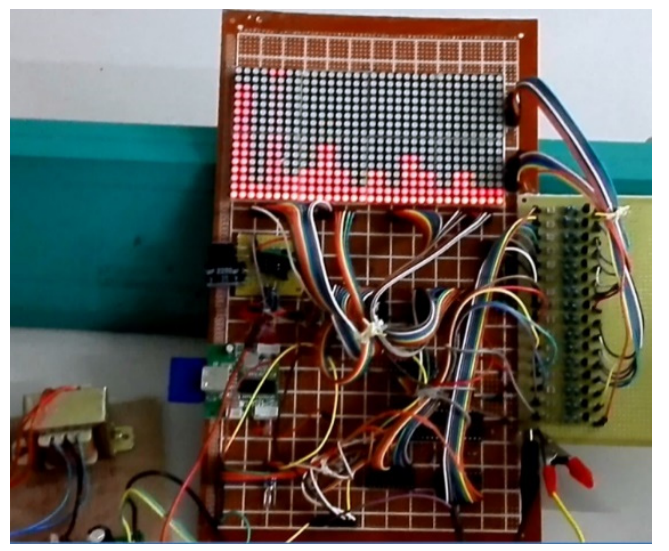

Figure 10. the overall effect of the system

\section{Conclusion}

The spectrum display realized by using the FFT overcomes the disadvantages of high hardware cost and complexity of hardware and software of the traditional audio display. After debugging, the frequency spectrum monitor shows that it is faster run and clearer sound, and the audio signal shows better following performance, and the audio signal can be adjusted automatically, and the audio signal can be automatically adjusted to the brightness. 


\section{References}

[1]. ZHANG Xiao-hu. DIY music spectrum display [J]. Electronic production.2011, (11): 38 - 44.

[2]. ZHOU Jin-rong, FENG Han-hui. The Design of DSP spectrum analysis system [J]. Journal of Heilongjiang Bayi Agricultural University.2013, 25 (5): 74 - 78.

[3]. WU Yong-de, LOU Ping. The Design of Music Spectrum Display Based on Enhanced 8051 MCU [J]. Electronic world, 2014, (13): 156 - 159. 